\title{
RESEARCH
}

Open Access

\section{Progression to dementia in memory clinic patients with mild cognitive impairment and normal $\beta$-amyloid}

Anna Rosenberg ${ }^{1 *}$ (D) Alina Solomon ${ }^{1,2}$, Vesna Jelic ${ }^{2,3}$, Göran Hagman ${ }^{2,3}$, Nenad Bogdanovic ${ }^{2,3}$ and Miia Kivipelto $2,3,4,5$

\begin{abstract}
Background: Determination of $\beta$-amyloid (A $\beta$ ) positivity and likelihood of underlying Alzheimer's disease (AD) relies on dichotomous biomarker cut-off values. Individuals with mild cognitive impairment $(\mathrm{MCl})$ and $A \beta$ within the normal range may still have a substantial risk of developing dementia, primarily of Alzheimer type. Their prognosis, as well as predictors of clinical progression, are not fully understood. The aim of this study was to explore the associations of cerebrospinal fluid (CSF) biomarkers (Aß42, total tau, phosphorylated tau) and other characteristics, including modifiable vascular factors, with the risk of progression to dementia among patients with $\mathrm{MCl}$ and normal CSF Aß42.
\end{abstract}

Methods: Three hundred eighteen memory clinic patients with CSF and clinical data, and at least 1-year follow-up, were included. Patients had normal CSF A 42 levels based on clinical cut-offs. Cox proportional hazard models with age as time scale and adjusted for sex, education, and cognition (Mini-Mental State Examination) were used to investigate predictors of progression to dementia and Alzheimer-type dementia. Potential predictors included CSF biomarkers, cognitive performance (verbal learning and memory), apolipoprotein $E(A P O E)$ \&4 genotype, medial temporal lobe atrophy, family history of dementia, depressive symptoms, and vascular factors, including the Cardiovascular Risk Factors, Aging and Dementia (CAIDE) risk score. Predictive performance of patient characteristics was further explored with Harrell C statistic.

Results: Lower normal $A \beta 42$ and higher total tau and phosphorylated tau were associated with higher dementia risk, and the association was not driven by A 442 values close to cut-off. Additional predictors included poorer cognition, APOE $\varepsilon 4$ genotype, higher systolic blood pressure, and lower body mass index, but not the CAIDE dementia risk score. Aß42 individually and in combination with other CSF biomarkers improved the risk prediction compared to age and cognition alone. Medial temporal lobe atrophy or vascular factors did not increase the predictive performance.

Conclusions: Possibility of underlying AD pathology and increased dementia risk should not be ruled out among MCI patients with CSF Aß42 within the normal range. While cut-offs may be useful in clinical practice to identify high-risk individuals, personalized risk prediction tools incorporating continuous biomarkers may be preferable among individuals with intermediate risk. The role of modifiable vascular factors could be explored in this context.

Keywords: Mild cognitive impairment, Alzheimer's disease, Dementia, Disease progression, Prognosis, Biomarkers, Cerebrospinal fluid

\footnotetext{
* Correspondence: anna.rosenberg@ueffi

${ }^{1}$ Department of Neurology, Institute of Clinical Medicine, University of

Eastern Finland, Kuopio, Finland

Full list of author information is available at the end of the article
}

(c) The Author(s). 2019 Open Access This article is distributed under the terms of the Creative Commons Attribution 4.0 International License (http://creativecommons.org/licenses/by/4.0/), which permits unrestricted use, distribution, and reproduction in any medium, provided you give appropriate credit to the original author(s) and the source, provide a link to the Creative Commons license, and indicate if changes were made. The Creative Commons Public Domain Dedication waiver (http://creativecommons.org/publicdomain/zero/1.0/) applies to the data made available in this article, unless otherwise stated. 


\section{Background}

Mild cognitive impairment (MCI) is a heterogeneous condition characterized by subjective cognitive complaints and objectively measured mild impairment in at least one cognitive domain [1]. The cumulative risk of progression to dementia in $\mathrm{MCI}$ has been estimated to range from approximately $22 \%$ (community-based studies) to 39\% (memory clinics), with the majority of individuals developing Alzheimer-type dementia [2]. To accurately identify individuals with underlying Alzheimer's disease (AD) early, at the MCI or even asymptomatic stage, several sets of diagnostic research criteria were proposed [3-9]. All criteria build upon biomarkers of AD neuropathology, combining them in different ways to classify individuals based on the probability of AD [3-9]. $\beta$-amyloid 1-42 (A $\beta 42)$ and its deposition in insoluble plaques in the brain is considered a pathological hallmark of $\mathrm{AD}$ [10]. Thus, diagnostic research criteria for $\mathrm{AD}$ underline the importance of biomarkers reflecting the accumulation of $A \beta 42$, namely decreased levels of $A \beta 42$ in the cerebrospinal fluid (CSF) and increased uptake of $A \beta 42$-specific tracers in positron emission tomography (PET) [3-9]. The most recent criteria, the National Institute on Aging-Alzheimer's Association (NIA-AA) Research Framework, propose that amyloid positivity, regardless of cognitive performance or other biomarker evidence, could be sufficient for classifying a person as being "in the Alzheimer's continuum" [9].

Determination of amyloid positivity currently relies on cut-off values. CSF cut-offs used in clinical practice vary between laboratories/clinics, and they are generally based on comparisons between healthy individuals and those with AD dementia diagnosis. Other cut-offs have been proposed by, e.g., examining the concordance of CSF A 42 with amyloid PET [11] or utilizing datadriven modeling [12]. Nevertheless, the amyloid positive versus negative dichotomy may not capture the full continuum of $\mathrm{AD}$ and dementia risk. In fact, $10-40 \%$ of MCI individuals with normal CSF A 42 may ultimately develop AD dementia [13-17], and CSF A $\beta 42$, as well as the other core $\mathrm{AD}$ biomarkers total tau (t-tau) and phosphorylated tau (p-tau), have been associated with an increased risk of progression to $\mathrm{AD}$ dementia among memory clinic patients with normal levels of CSF A 442 [18].

As studies are scarce, and previous findings may not always be generalizable to other populations or settings, more evidence is needed for the role of CSF A 342 and other biomarkers in predicting progression to dementia among individuals with CSF A $\beta 42$ within the normal range. Moreover, it is not fully clear if other characteristics, such as modifiable vascular and lifestyle-related factors, are associated with the risk of progression in $\mathrm{MCI}$ patients with normal A $\beta 42$ levels. Such information might help identify a window of opportunity for secondary prevention in this patient population and inform lifestylebased and vascular prevention trials-which may not select subjects based on their amyloid status-of potential new targets.

The aim of this study was to investigate whether (1) levels of CSF A $\beta 42$, t-tau, and p-tau and (2) other characteristics, including modifiable vascular factors, were associated with the risk of progression to dementia/AD dementia among memory clinic patients with $\mathrm{MCI}$ and normal CSF A $\beta 42$ levels.

\section{Methods}

\section{Study population}

The study included 318 patients diagnosed with MCI at the Karolinska University Hospital memory clinic in Huddinge, Sweden, during 2007-2014, who consented to have their data included in the clinic's research database. Criteria used to identify individuals in the database for the present study were as follows: at least 1 year of follow-up, availability of baseline CSF and other data relevant for the study, and normal CSF A 42 levels based on the cut-offs employed at the clinic. The study was approved by the Regional Ethical Review Board in Stockholm, and written informed consent was obtained from all patients.

Routine assessments at the memory clinic consisted of a physical and neurological examination, thorough review of medical history, Mini-Mental State Examination (MMSE) [19] and comprehensive neuropsychological testing, routine blood tests, CSF sampling to measure AD biomarkers $(\mathrm{A} \beta 42, \mathrm{t}$-tau, $\mathrm{p}$-tau), brain imaging (structural magnetic resonance imaging, MRI or computed tomography, CT), and other assessments, depending on the patient's clinical presentation [20]. Diagnoses were made on average within 2 months from the beginning of the assessment period by consensus in multidisciplinary meetings of the clinic staff using all available clinical data, including CSF biomarker data, in an unblinded manner. MCI was diagnosed using the consensus criteria for $\mathrm{MCI}$ which require the presence of both subjective and objective cognitive impairment involving one or more cognitive domains, but no impairment of activities of daily living and no dementia [21]. Objective cognitive impairment was defined as a test performance of $1.5 \mathrm{SD}$ below what is expected based on age and education. Dementia diagnoses were made according to the Diagnostic and Statistical Manual of Mental Disorders, $4^{\text {th }}$ edition (DSM-IV) [22] criteria, and etiology was diagnosed using the National Institute of Neurological and Communicative Disorders and StrokeAlzheimer's Disease and Related Disorders Association (NINCDS-ADRDA) criteria by McKhann et al. [23] for $A D$, National Institute of Neurological Disorders and Stroke-Association Internationale pour la Recherche et l'Enseignement en Neurosciences (NINDS-AIREN) [24] 
criteria for vascular dementia, criteria by Neary et al. [25] for frontotemporal dementia, and the Movement Disorder Society Task Force [26] criteria for Parkinson's disease dementia, as previously reported $[27,28]$. The necessity and frequency of follow-up visits were based on the clinician's judgment as per local routine clinical practice. Follow-up data in the present study were collected until April 2018. Main outcome in this study was progression to any dementia, and progression to $\mathrm{AD}$ dementia was also investigated.

\section{CSF biomarkers}

CSF samples were collected by standard lumbar puncture done between the L3/L4 or L4/L5 intervertebral space with a 25 -gauge needle. Samples were collected in polypropylene tubes and centrifuged within $2 \mathrm{~h}$. CSF $\mathrm{A} \beta 42$, $\mathrm{t}$-tau, and $\mathrm{p}$-tau concentrations were measured with commercially available sandwich enzyme-linked immunosorbent assays (Innogenetics, Ghent, Belgium) [29]. CSF A 342 positivity was determined based on the reference values provided by the laboratories conducting the analyses, and employed in clinical practice at the memory clinic, as previously reported [27, 30-32] (cutoff $<450 \mathrm{pg} / \mathrm{ml}$ defined by the Karolinska University Hospital in Stockholm, Sweden, until 2011; cut-off < $550 \mathrm{pg} / \mathrm{ml}$ defined by the Clinical Neurochemistry Laboratory at Sahlgrenska University Hospital in Gothenburg, Sweden, from 2012 onwards). Patients with $A \beta 42$ levels $\geq 450 \mathrm{pg} / \mathrm{ml}$ (first visit during 2007-2011) or $\geq$ $550 \mathrm{pg} / \mathrm{ml}$ (first visit in 2012 or later) were considered to have normal CSF A $\beta 42$ levels. Cut-off values for abnormal t-tau and p-tau were $\geq 400 \mathrm{pg} / \mathrm{ml}$ and $\geq 80 \mathrm{pg} / \mathrm{ml}$, respectively [30].

As there is increasing evidence suggesting that the currently used clinical cut-offs for CSF A $\beta 42$ positivity may be too conservative due to, e.g., upward drift in $A \beta 42$ values over time in certain assays [33, 34], and more lenient cut-offs may predict underlying amyloid pathology more accurately $[11,12]$, we performed sensitivity analyses in a subsample of patients with CSF A 342 levels $>696 \mathrm{pg} /$ $\mathrm{ml}(N=195)$. This cut-off for MCI patients was proposed by Bertens et al. [12] in a Dutch memory clinic population using a data-driven approach. The Dutch clinical cut-off, $550 \mathrm{pg} / \mathrm{ml}$, was comparable to the one used at the Karolinska University Hospital memory clinic.

\section{MRI and CT assessment}

Brain MRI or CT scans were performed in connection to the first visit at the memory clinic or as part of the general practitioner's initial assessment prior to the referral. MRI visual assessments were performed based on T1-weighted images, and medial temporal lobe atrophy (MTA) was rated using the Scheltens scale [35] ranging from 0 (no atrophy) to 4 (severe atrophy). Where applicable, MTA rating was performed also on the CT scans (54 of the 237 patients with available brain imaging data). MTA was visually assessed for right and left hemisphere separately, and the mean score was used in the present study.

\section{APOE genotyping}

DNA was extracted from blood leucocytes using standard methods polymerase chain reaction and HhaI digestion, and apolipoprotein E (APOE) genotype was determined by a microsequencing method on microtiter plates (AffiGene ApoE, Sangtec Medical, Bromma, Sweden), as previously described $[29,30]$.

\section{Other clinical characteristics}

Patients' demographic and clinical characteristics, including age, sex, years of formal education, cognitive test scores (MMSE, Rey Auditory Verbal Learning Test (RAVLT) immediate and delayed recall [36]), and information about medications and medical history (hypertension, hyperlipidemia, diabetes) were obtained from medical records from the baseline visit at the memory clinic. Additional data collected from the medical records included the presence of depressive symptoms (measured on Cornell Scale for Depression in Dementia [37]), systolic and diastolic blood pressure, smoking habits, and height and weight. Body mass index (BMI) was calculated by dividing the weight in kilograms by the squared height in meters. Self-reported family history of any type of dementia was considered positive when there was at least one affected first-degree relative.

The Cardiovascular Risk Factors, Aging and Dementia (CAIDE) risk score [38] was calculated using demographic and clinical data collected from the medical records (Additional file 1: Table S1). As data about lifestyle, including physical activity, were not routinely collected at the memory clinic, the physical activity component was excluded from the CAIDE risk score [39]. For elevated total cholesterol, two points were given if the patient had hyperlipidemia (diagnosis and treatment with any lipid-lowering drug). For elevated systolic blood pressure (SBP), two points were given if the patient either had hypertension (diagnosis and treatment with any antihypertensive drug), or SBP measured at the first visit was elevated (>140 $\mathrm{mmHg}$ ). The CAIDE risk score version used in the present study ranged from 0 to 14 (version with $A P O E$ 0-17), with higher values indicating higher risk of dementia.

\section{Statistical analysis}

Baseline demographic and clinical characteristics were compared between patients who progressed to dementia and those who did not with $t$ tests and chi-square tests, as appropriate. Associations between individual baseline 
characteristics and risk of progression to dementia/AD dementia were analyzed with Cox proportional hazard models with age as time scale. Models included additionally sex, education, and baseline MMSE as covariates. Results are reported as hazard ratios (HR) and $95 \%$ confidence intervals (CI). Zero-skewness log-transformation followed by $\mathrm{z}-$ transformation was applied to CSF biomarkers to obtain hazard ratios per SD and to compare the associations with dementia/AD dementia between different biomarkers.

We further estimated the predictive performance (Harrell C statistic) of all individual baseline characteristics showing significant associations with the risk of progression to any dementia in the univariate analysis $(p<$ 0.10 ). Harrell $C$ statistic of 0.5 indicates no predictive value, whereas 1.0 indicates complete prediction, on a scale from 0 to 1 . We compared the Harrell $\mathrm{C}$ statistic of a basic model including only age and cognitive performance (RAVLT delayed recall score) with models expanded with additional predictors. All analyses were conducted with Stata software version 14, and level of statistical significance was set to $p<0.05$.

\section{Results}

Baseline characteristics of the study population are shown in Table 1. Patients' mean age was 64.8 years, and 174 (54.7\%) were women. Patients had on average 11.7 years of education, and their mean MMSE score was 27.1 points. In total, $50.3 \%$ (86 out of 171 ) of the patients with available $A P O E$ genotype data were $\varepsilon 4$ carriers, and $38.9 \%$ (119 out of 306) had a family history of dementia. CSF $\mathrm{t}$-tau and $\mathrm{p}$-tau levels were considered abnormal in $92(29.0 \%)$ and $60(18.9 \%)$ patients, respectively. Of the 236 patients for whom all biomarkers were available, 135 (57.2\%) could be classified as having evidence for neurodegeneration in the absence of definitely abnormal $\mathrm{A} \beta$, i.e., at least one abnormal marker of neurodegeneration (CSF t-tau, p-tau, MTA).

During a mean follow-up period of 2.8 years (SD 1.9, range $1-10$ years), 121 patients (38.1\%) progressed to any dementia, with most cases being AD dementia $(N=91)$. Other dementias included unspecified dementia $(N=11)$, vascular dementia $(N=10)$, frontotemporal dementia $(N=6)$, and Parkinson's disease dementia $(N=3)$. Patients with evidence for neurodegeneration progressed to dementia more often than those with normal biomarkers of neurodegeneration (70 out of 135; 51.9\% vs. 26 out of 101 ; $25.7 \%, p<0.001)$. Differences in baseline characteristics between patients who progressed to dementia and patients who remained stable or reverted to normal are presented in (Table 1). Patients progressing to dementia were significantly older and performed worse in verbal learning and memory test (RAVLT) than patients who did not show clinical progression. Furthermore, they had significantly lower, but still normal, CSF A $\beta 42$ levels, higher t-tau and p-tau levels, higher MTA score, and lower Cornell score, indicating fewer depressive symptoms. Patients who progressed to dementia were also more often APOE \&4 carriers. Vascular risk profile of converters and nonconverters was similar, except for SBP which was higher among patients who developed dementia. Similar baseline differences were observed between patients who progressed to $\mathrm{AD}$ dementia and those who did not progress to any dementia (Additional file 1: Table S2).

\section{CSF biomarkers and risk of progression to dementia/AD dementia}

Associations of CSF biomarkers with the risk of progression to dementia are presented in Table 2 . Among patients with normal CSF A $\beta 42$ levels, higher A $\beta 42$ (HR 0.65, 95\% CI 0.52-0.81) was related to a lower risk of dementia, while higher t-tau (HR 2.16, 95\% CI 1.70-2.74) and p-tau (HR 1.53 , 95\% CI 1.25-1.89) were associated with a higher risk of progression to any dementia. Higher $A \beta 42 / t$-tau and A $342 / p$-tau ratios were associated with a lower risk of dementia. Similar significant associations were observed in analyses with $\mathrm{AD}$ dementia as outcome (Table 2), and in sensitivity analyses including patients classified as having normal CSF A $\beta 42$ levels based on a higher cut-off for amyloid positivity (>696 pg/ml) (Table 3). Of the three CSF biomarkers, t-tau levels showed the highest HRs for progression to any dementia and $\mathrm{AD}$ dementia.

\section{Other characteristics and risk of progression to dementia/ AD dementia}

Associations of other patient characteristics with the risk of progression to dementia are presented in Table 4 . Having lower RAVLT immediate and delayed recall test scores (HR 0.94, 95\% CI 0.91-0.96 and HR 0.81, 95\% CI $0.75-0.88$, respectively) and being $A P O E \varepsilon 4$ carrier (HR 2.03, 95\% CI 1.18-3.46) were associated with a higher risk of dementia. Depressive symptoms were associated with a lower risk of progression (HR 0.94, 95\% CI 0.890.99). Having an MTA score $>1$ showed a borderline significant association with dementia risk (HR 1.53, 95\% CI 0.99-2.36). Among vascular factors, higher SBP (HR 1.02, 95\% CI 1.01-1.03) and lower BMI (HR 0.93, 95\% CI 0.86-0.99) were significantly associated with a higher dementia risk. CAIDE dementia risk score, with or without $A P O E$, was not associated with an increased dementia risk in this patient population. Similar results were obtained from analyses with AD dementia as outcome (Table 4). However, more pronounced MTA was not significantly associated with risk of progression to $\mathrm{AD}$ dementia (HR 1.45, 95\% CI 0.88-2.39), and lower BMI showed only a borderline significant association (HR 0.93, 95\% CI 0.85-1.00). Associations of patient characteristics with the risk of progression to dementia among 
Table 1 Baseline characteristics of the study population, by outcome (progression to any dementia)

\begin{tabular}{|c|c|c|c|c|c|}
\hline Characteristics & Data available & All $(N=318)$ & Progression to dementia $(N=121)$ & No progression to dementia $(N=197)$ & $P$ value \\
\hline \multicolumn{6}{|l|}{ Demographics } \\
\hline Age, years & 318 & $64.8(9.1)$ & $67.9(8.3)$ & $63.0(9.1)$ & $<0.001$ \\
\hline Female & 318 & $174(54.7 \%)$ & $64(52.9 \%)$ & $110(55.8 \%)$ & 0.61 \\
\hline Education, years & 293 & $11.7(3.7)$ & $11.7(3.5)$ & $11.7(3.9)$ & 0.97 \\
\hline Follow-up time, years & 318 & $2.8(1.9)$ & $2.9(1.9)$ & $2.8(1.9)$ & 0.83 \\
\hline \multicolumn{6}{|l|}{ Cognition } \\
\hline MMSE score & 315 & $27.1(2.5)$ & $27.0(2.3)$ & $27.1(2.7)$ & 0.72 \\
\hline RAVLT, immediate recall score & 215 & $34.6(9.8)$ & $30.6(8.4)$ & $37.5(9.7)$ & $<0.001$ \\
\hline RAVLT, delayed recall score & 235 & $5.6(3.3)$ & $4.2(2.9)$ & $6.5(3.3)$ & $<0.001$ \\
\hline \multicolumn{6}{|l|}{ Biomarkers } \\
\hline CSF A $342, \mathrm{pg} / \mathrm{ml}$ & 318 & $849.1(293.6)$ & $703.7(222.5)$ & $938.5(296.7)$ & $<0.001$ \\
\hline CSF t-tau, pg/ml & 317 & $335.5(184.9)$ & $418.0(193.5)$ & $284.5(159.7)$ & $<0.001$ \\
\hline CSF p-tau, pg/ml & 317 & $58.0(25.1)$ & $65.7(22.8)$ & $53.3(25.4)$ & $<0.001$ \\
\hline Abnormal t-tau ( $\geq 400$ pg/ml) & 317 & $92(29.0 \%)$ & $55(45.5 \%)$ & $37(18.9 \%)$ & $<0.001$ \\
\hline Abnormal p-tau ( $\geq 80$ pg/ml) & 317 & $60(18.9 \%)$ & $31(25.6 \%)$ & $29(14.8 \%)$ & 0.02 \\
\hline CSF A $342 /$ t-tau ratio & 317 & $3.4(2.0)$ & $2.2(1.4)$ & $4.1(2.0)$ & $<0.001$ \\
\hline CSF A $342 / p$-tau ratio & 317 & $18.0(10.4)$ & $12.5(6.9)$ & $21.4(10.7)$ & $<0.001$ \\
\hline MTA score & 237 & $1.1(0.8)$ & $1.3(0.7)$ & $1.0(0.8)$ & 0.001 \\
\hline MTA score > 1 & 237 & $84(35.4 \%)$ & $45(46.9 \%)$ & $39(27.7 \%)$ & 0.002 \\
\hline \multicolumn{6}{|l|}{ Vascular factors } \\
\hline Systolic BP, mmHg & 245 & $143.5(19.2)$ & $148.4(21.7)$ & $140.6(17.1)$ & 0.002 \\
\hline Diastolic BP, mmHg & 245 & $83.4(10.5)$ & $84.7(10.5)$ & $82.6(10.5)$ & 0.13 \\
\hline $\mathrm{BMI}, \mathrm{kg} / \mathrm{m}^{2}$ & 205 & $26.0(4.0)$ & $25.5(4.0)$ & $26.3(4.0)$ & 0.23 \\
\hline Current smoking & 287 & $41(14.3 \%)$ & $15(13.5 \%)$ & $26(14.8 \%)$ & 0.77 \\
\hline Hypertension & 318 & 130 (40.9\%) & $54(44.6 \%)$ & $76(38.6 \%)$ & 0.29 \\
\hline Hyperlipidemia & 318 & $93(29.3 \%)$ & $40(33.1 \%)$ & $53(26.9 \%)$ & 0.24 \\
\hline Diabetes & 318 & $53(16.7 \%)$ & $20(16.5 \%)$ & $33(16.8 \%)$ & 0.96 \\
\hline CAIDE risk score & 174 & $7.5(2.5)$ & $7.9(2.1)$ & $7.4(2.6)$ & 0.18 \\
\hline CAIDE risk score with $A P O E$ & 91 & $9.4(2.4)$ & $9.8(2.4)$ & $9.2(2.4)$ & 0.22 \\
\hline \multicolumn{6}{|l|}{ Other factors } \\
\hline APOE \&4 carrier & 171 & $86(50.3 \%)$ & $44(65.7 \%)$ & $42(40.4 \%)$ & 0.001 \\
\hline Family history of dementia & 306 & $119(38.9 \%)$ & 47 (39.8\%) & $72(38.3 \%)$ & 0.79 \\
\hline Cornell score & 248 & $6.1(4.7)$ & $5.2(4.2)$ & $6.6(4.9)$ & 0.02 \\
\hline
\end{tabular}

Data are mean (SD) or $N$ (\%). $P$ values are shown for comparisons between patients who developed/did not develop dementia. $P$ values $<0.05$ are italicized. Hypertension and hyperlipidemia were defined as diagnosis of hypertension/hyperlipidemia and treatment with any antihypertensive/lipid-lowering drug. Family history of dementia included at least one affected first-degree relative. APOE apolipoprotein $\mathrm{E}, A \beta 42 \beta$-amyloid $1-42, B M I$ body mass index, $B P$ blood pressure, CAIDE Cardiovascular Risk Factors, Aging and Dementia Study, CSF Cerebrospinal fluid, MMSE Mini-Mental State Examination, MTA medial temporal lobe atrophy, visual rating, RAVLT Rey Auditory Verbal Learning Test, $p$-tau tau phosphorylated at threonine 181, $t$-tau total tau

patients with CSF $\mathrm{A} \beta 42>696 \mathrm{pg} / \mathrm{ml}$ are presented in Table 5.

\section{Predictive performance of CSF biomarkers and other patient characteristics}

Predictive performance of the basic model (age and cognition) and models expanded with additional other predictors (CSF A $\beta 42$ individually and in combination with other CSF biomarkers, MTA, APOE, depressive symptoms, SBP, BMI) are shown in Table 6. Harrell C statistic for the basic model ranged from 0.68 to 0.72 , depending on the number of patients included in each model (with variations due to missing data). Adding $A \beta 42$ increased Harrell $C$ from 0.69 to 0.73 . Together with $\mathrm{A} \beta 42$, $\mathrm{p}$-tau and $\mathrm{t}$-tau further improved predictive performance (Harrell C 0.75 for p-tau, 0.74 for $\mathrm{A} \beta 42 / \mathrm{p}$-tau ratio, 0.76 for $\mathrm{t}$-tau and $\mathrm{A} \beta 42 / \mathrm{t}$-tau ratio, and 0.78 for all three CSF biomarkers). Similar results were obtained in 
Table 2 Association of CSF biomarkers with the risk of progression to any dementia/AD dementia

\begin{tabular}{lll}
\hline CSF biomarker & Progression to any dementia, HR (95\% Cl) & Progression to AD dementia, HR (95\% Cl) \\
\hline A 342 & $0.65(0.52-0.81)$ & $0.55(0.42-0.72)$ \\
t-tau & $2.16(1.70-2.74)$ & $3.28(2.42-4.46)$ \\
p-tau & $1.53(1.25-1.89)$ & $1.99(1.55-2.56)$ \\
A $342 /$ t-tau & $0.45(0.35-0.57)$ & $0.28(0.20-0.40)$ \\
A $\beta 42 /$ p-tau & $0.56(0.45-0.70)$ & $0.41(0.31-0.54)$ \\
\hline
\end{tabular}

HR (hazard ratios) $(95 \% \mathrm{Cl})$ per 1 SD increase in CSF biomarkers are shown from Cox proportional hazard models with age as time scale. Models were adjusted for sex, education, and baseline MMSE score. AD Alzheimer's disease, Aß42 $\beta$-amyloid 1-42, CSF cerebrospinal fluid, $p$-tau tau phosphorylated at threonine 181, $t$-tau total tau

sensitivity analyses using a more lenient cut-off for amyloid positivity (Table 7): adding A $\beta 42$ alone to the basic model increased Harrell $C$ from 0.74 to 0.79 , and the model including all three CSF biomarkers had the highest predictive performance (0.83).

$A P O E \& 4$ genotype increased the predictive performance of the basic model (increase in Harrell $C$ from 0.68 to 0.72 ), similarly to $A \beta 42$, but depressive symptoms, MTA, or vascular factors did not substantially improve the predictive performance of the basic model (Table 6). Similar results were obtained with $\mathrm{AD}$ dementia as outcome.

\section{Discussion}

This study explored the associations of CSF AD biomarkers and other patient characteristics with progression to dementia among memory clinic patients with MCI and normal CSF A $\beta 42$ levels. Lower $A \beta 42$ levels within the normal range, as well as higher $\mathrm{t}$-tau and p-tau levels, were significantly associated with a higher risk of progression to any dementia and AD dementia in this population. Additional predictors were poorer cognitive performance, $A P O E \& 4$ genotype, higher SBP, and lower BMI. No association was observed between the CAIDE dementia risk score and risk of progression. $A \beta 42$, both alone and in combination with t-tau and $\mathrm{p}$-tau, improved the prediction of progression to dementia compared to age and cognitive performance alone, whereas vascular factors did not substantially increase the predictive performance.
According to the current diagnostic research criteria for AD [5-9], patients with $\mathrm{MCI}$ and normal CSF A 442 levels would be assumed to have an underlying non- $A D$ pathology. A range of non-AD-related processes, such as primary age-related tauopathy, hippocampal sclerosis, cerebrovascular disease, argyrophilic grain disease, and TDP-43 encephalopathy, could contribute to neurodegeneration in this population [40-42]. While in previous studies the rate of clinical progression was consistently lower among MCI patients with evidence for neurodegeneration in the absence of definitely abnormal amyloid than among those with abnormal markers of both amyloid and neurodegeneration, a significant number of individuals still developed $\mathrm{AD}$ dementia during a fairly short follow-up period [13-17]. In line with these findings, we observed that, despite having normal CSF A $\beta 42$ levels, a high proportion of $\mathrm{MCI}$ patients progressed to dementia (primarily of Alzheimer type).

Our results indicating that lower $A \beta 42$ levels within the normal range were significantly associated with an increased risk of progression are also supported by previous findings [18]. It has been unclear whether the effect is primarily driven by $A \beta 42$ values just above the cut-off value [13, 18]; however, we observed a similar pattern in a sensitivity analysis with a more lenient cutoff value for amyloid positivity. This suggests that simply increasing cut-off values might not be sufficient to optimally distinguish future converters from individuals who remain stable or develop non-AD dementia. As the clinical impact of amyloid accumulation and threshold for cognitive decline and disease progression may vary

Table 3 Association of CSF biomarkers with the risk of progression to any dementia/AD dementia (patients with CSF Aß42 > 696 $\mathrm{pg} / \mathrm{ml})$

\begin{tabular}{lll}
\hline CSF biomarker & Progression to any dementia, HR $(95 \% \mathrm{Cl})$ & Progression to AD dementia, HR (95\% Cl) \\
\hline A 342 & $0.58(0.39-0.87)$ & $0.55(0.33-0.93)$ \\
t-tau & $2.36(1.64-3.38)$ & $4.33(2.52-7.44)$ \\
p-tau & $1.43(1.04-1.96)$ & $1.99(1.28-3.08)$ \\
A $342 /$ t-tau & $0.38(0.25-0.56)$ & $0.21(0.11-0.38)$ \\
A $342 /$ p-tau & $0.55(0.39-0.79)$ & $0.37(0.22-0.62)$ \\
\hline
\end{tabular}

HR (hazard ratios) $(95 \% \mathrm{CI})$ per 1 SD increase in CSF biomarkers are shown from Cox proportional hazard models with age as time scale. Models were adjusted for sex, education, and baseline MMSE score. AD Alzheimer's disease, $A \beta 42 \beta$-amyloid $1-42$, CSF cerebrospinal fluid, $p$-tau tau phosphorylated at threonine $181, t$-tau total tau 
Table 4 Association of cognition, vascular factors, and other characteristics with the risk of progression to any dementia/AD dementia

\begin{tabular}{lll}
\hline Characteristics & HR (95\% Cl) & \\
\cline { 2 - 3 } & Any dementia & AD dementia \\
\hline Cognition & & \\
RAVLT, immediate recall score & $0.94(0.91-0.96)$ & $0.94(0.91-0.97)$ \\
RAVLT, delayed recall score & $0.81(0.75-0.88)$ & $0.82(0.74-0.90)$ \\
Vascular factors & & \\
Systolic BP, mmHg & $1.02(1.01-1.03)$ & $1.01(1.00-1.02)$ \\
Diastolic BP, mmHg & $1.01(0.99-1.03)$ & $1.00(0.97-1.02)$ \\
BMI, kg/m ${ }^{2}$ & $0.93(0.86-0.99)$ & $0.93(0.85-1.00)$ \\
Current smoking & $0.96(0.54-1.69)$ & $1.05(0.57-1.95)$ \\
Hypertension & $1.19(0.81-1.75)$ & $1.01(0.64-1.59)$ \\
Hyperlipidemia & $0.98(0.65-1.47)$ & $0.90(0.56-1.45)$ \\
Diabetes & $0.74(0.44-1.25)$ & $0.70(0.38-1.31)$ \\
CAIDE risk score & $1.00(0.88-1.14)$ & $0.97(0.83-1.13)$ \\
CAIDE risk score with APOE & $1.11(0.91-1.35)$ & $1.07(0.87-1.33)$ \\
Other factors & & \\
MTA score > 1 & $1.53(0.99-2.36)$ & $1.45(0.88-2.39)$ \\
APOE \&4 genotype & $2.03(1.18-3.46)$ & $2.50(1.36-4.61)$ \\
Family history of dementia & $1.00(0.67-1.48)$ & $1.04(0.66-1.62)$ \\
Cornell score & $0.94(0.89-0.99)$ & $0.90(0.85-0.96)$ \\
\hline HR (hazard ratios) $95 \%$ CI) are shown fom Cox propotiona & hazad modes
\end{tabular}

HR (hazard ratios) $(95 \% \mathrm{Cl})$ are shown from Cox proportional hazard models with age as time scale. Models were adjusted for sex, education, and baseline MMSE score. Hypertension and hyperlipidemia were defined as diagnosis of hypertension/hyperlipidemia and treatment with any antihypertensive/lipidlowering drug. Family history of dementia included at least one affected firstdegree relative. $A D$ Alzheimer's disease, $A P O E$ apolipoprotein $E, B M I$ body mass index, $B P$ blood pressure, CAIDE Cardiovascular Risk Factors, Aging and Dementia Study, MTA medial temporal lobe atrophy, visual rating, RAVLT Rey Auditory Verbal Learning Test

among individuals, due to, e.g., cognitive reserve [43] or mixed pathologies (e.g., vascular) [44], treating biomarkers as continuous may be preferred in certain subgroups of MCI patients. A PET study by Farrell et al. [45] showed that continuous measures reflecting amyloid deposition may provide more detailed information about the predicted rate of cognitive decline than the dichotomized amyloid status. The study focused, however, on cognitively healthy individuals and change in cognition, rather than clinical progression. Also, the effect appeared to be limited to amyloid-positive individuals. Among amyloid-negative individuals, preliminary evidence from recent longitudinal PET studies suggests that amyloid accumulation is associated with cognitive decline [46, 47], whereas baseline amyloid burden is not [46]. Again, these studies did not investigate clinical progression as an outcome, but Landau et al. [47] reported the numbers of individuals who converted to amyloid positive and progressed to $\mathrm{MCI}$ or $\mathrm{AD}$. Interestingly, very little overlap was observed between these groups.
Table 5 Association of cognition, vascular factors, and other characteristics with the risk of progression to any dementia/AD dementia (patients with CSF Aß42> 696 pg/ml)

\begin{tabular}{lll}
\hline Characteristics & HR $(95 \%$ Cl & \\
\cline { 2 - 3 } & Any dementia & AD dementia \\
\hline Cognition & & \\
RAVLT, immediate recall score & $0.92(0.88-0.97)$ & $0.93(0.88-0.99)$ \\
RAVLT, delayed recall score & $0.79(0.69-0.91)$ & $0.84(0.72-0.99)$ \\
Vascular factors & & \\
Systolic BP, mmHg & $1.03(1.01-1.05)$ & $1.02(0.99-1.04)$ \\
Diastolic BP, mmHg & $1.01(0.98-1.05)$ & $0.98(0.94-1.03)$ \\
BMI, kg/m ${ }^{2}$ & $0.89(0.80-0.99)$ & $0.95(0.82-1.11)$ \\
Current smoking & $1.21(0.46-3.18)$ & $1.29(0.37-4.52)$ \\
Hypertension & $1.28(0.69-2.39)$ & $1.34(0.60-2.97)$ \\
Hyperlipidemia & $0.87(0.45-1.68)$ & $0.51(0.21-1.26)$ \\
Diabetes & $0.68(0.31-1.46)$ & $0.89(0.36-2.20)$ \\
CAIDE risk score & $0.99(0.82-1.19)$ & $1.03(0.79-1.34)$ \\
CAIDE risk score with APOE & $1.29(0.86-1.93)$ & $1.49(0.81-2.75)$ \\
Other factors & & \\
MTA score > 1 & $2.21(1.09-4.45)$ & $2.60(1.08-6.29)$ \\
APOE \&4 genotype & $2.07(0.83-5.17)$ & $3.28(1.11-9.71)$ \\
Family history of dementia & $0.62(0.30-1.29)$ & $0.57(0.22-1.47)$ \\
Cornell score & $0.94(0.87-1.02)$ & $0.85(0.75-0.97)$ \\
\hline
\end{tabular}

HR (hazard ratios) $(95 \% \mathrm{Cl}$ ) are shown from Cox proportional hazard models with age as time scale. Models were adjusted for sex, education, and baseline MMSE score. Hypertension and hyperlipidemia were defined as diagnosis of hypertension/hyperlipidemia and treatment with any antihypertensive/lipidlowering drug. Family history of dementia included at least one affected firstdegree relative. $A D$ Alzheimer's disease, $A P O E$ apolipoprotein $E, A \beta 42 \beta$ amyloid 1-42, BMI body mass index, $B P$ blood pressure, CAIDE Cardiovascular Risk Factors, Aging and Dementia Study, CSF cerebrospinal fluid, MTA medial temporal lobe atrophy, visual rating, RAVLT Rey Auditory Verbal Learning Test

As these studies did not include patients with MCI, the magnitude and impact of change in amyloid levels at the MCI stage remains unclear.

In our MCI population with CSF A $\beta 42$ levels within the normal range, $A P O E$ \&4 showed similar predictive value to that of $\mathrm{A} \beta 42$ for short-term progression to dementia, suggesting that $A P O E$ genotyping could potentially be incorporated in the risk assessment in this patient population. $A P O E$ \&4 genotype has previously been linked to an increased risk of conversion from $\mathrm{MCI}$ to dementia, but studies have primarily focused on heterogeneous MCI populations, without stratifying by amyloid pathology $[48,49]$. CSF $\mathrm{t}$-tau and $\mathrm{p}$-tau were also associated with a higher risk of progression to dementia/AD dementia in our study. Similar findings were previously reported in patients with normal $A \beta 42$ levels and $\mathrm{MCI}$, but not subjective cognitive impairment [18]. As shown before, neuronal injury biomarkers predict disease progression and correlate well with cognitive deterioration among MCI patients [10]. In our study, 
Table 6 Harrell C statistic and model performance in prediction of any dementia/AD dementia

\begin{tabular}{|c|c|c|c|c|}
\hline \multirow[t]{2}{*}{ Categories of predictors } & \multirow[t]{2}{*}{$N$} & \multirow[t]{2}{*}{ Prediction model } & Dementia & AD dementia \\
\hline & & & \multicolumn{2}{|l|}{ Harrell C } \\
\hline \multirow[t]{7}{*}{ Basic model vs. CSF biomarkers } & \multirow[t]{7}{*}{234} & Age, RAVLT (basic) & 0.69 & 0.69 \\
\hline & & Age, RAVLT, Aß42 & 0.73 & 0.75 \\
\hline & & Age, RAVLT, Aß42, p-tau & 0.75 & 0.78 \\
\hline & & Age, RAVLT, Aß42/p-tau & 0.74 & 0.78 \\
\hline & & Age, RAVLT, Aß42, t-tau & 0.76 & 0.81 \\
\hline & & Age, RAVLT, Aß42/t-tau & 0.76 & 0.81 \\
\hline & & Age, RAVLT, Aß42, p-tau, t-tau & 0.78 & 0.83 \\
\hline \multirow[t]{2}{*}{ Basic model vs. MTA } & \multirow[t]{2}{*}{171} & Age, RAVLT (basic) & 0.71 & 0.72 \\
\hline & & Age, RAVLT, MTA & 0.71 & 0.72 \\
\hline \multirow[t]{2}{*}{ Basic model vs. APOE \&4 genotype } & \multirow[t]{2}{*}{133} & Age, RAVLT (basic) & 0.68 & 0.68 \\
\hline & & Age, RAVLT, APOE & 0.72 & 0.72 \\
\hline \multirow[t]{2}{*}{ Basic model vs. SBP } & \multirow[t]{2}{*}{182} & Age, RAVLT (basic) & 0.69 & 0.71 \\
\hline & & Age, RAVLT, SBP & 0.70 & 0.71 \\
\hline \multirow[t]{2}{*}{ Basic model vs. BMI } & \multirow[t]{2}{*}{141} & Age, RAVLT (basic) & 0.72 & 0.71 \\
\hline & & Age, RAVLT, BMl & 0.74 & 0.74 \\
\hline \multirow[t]{2}{*}{ Basic model vs. depressive symptoms } & \multirow[t]{2}{*}{183} & Age, RAVLT (basic) & 0.69 & 0.72 \\
\hline & & Age, RAVLT, Cornell score & 0.70 & 0.74 \\
\hline
\end{tabular}

Predictive performance of the prediction models was explored using the Harrell C statistic ( 0.5 indicates no predictive value, whereas 1.0 indicates complete prediction, on a scale from 0 to 1). Comparisons of model performance were conducted between models including the same number of patients: basic model (age, RAVLT delayed recall score) and models expanded with additional predictors. AD Alzheimer's disease, $A P O E$ apolipoprotein E, $A \beta 42 \beta$-amyloid 1-42, BMI body mass index; CSF cerebrospinal fluid, MTA medial temporal lobe atrophy, visual rating, $p$-tau tau phosphorylated at threonine 181, RAVLT Rey Auditory Verbal Learning Test (delayed recall), SBP systolic blood pressure, $t$-tau total tau

however, we did not observe a significant association between visually rated MTA and risk of progression to dementia, and MTA did not substantially increase the predictive performance of age and cognition alone. A measure of hippocampal volume, which may have more predictive value $[50,51]$, was not available for this study.

Among other patient characteristics, depressive symptoms were associated with a lower risk of dementia/AD dementia. Rather than reflecting a protective effect, our results may indicate that depression was a common underlying cause of MCI, as reported before [52]. Vascular factors, such as smoking, hypertension, hyperlipidemia, diabetes, and the CAIDE dementia risk score, were not associated with an increased risk of dementia in this MCI population with normal CSF A $\beta 42$ levels. Lower BMI and higher SBP increased the risk of dementia/AD dementia, but did not markedly improve the predictive performance compared to age and cognition alone. While vascular and lifestyle-related risk factors have been shown to increase the risk of subsequent dementia in midlife [53], their association with cognitive decline and dementia might be less straightforward at older ages and during shorter follow-ups [54, 55]. Factors such as blood pressure, BMI, or cholesterol have been reported to decline after midlife in individuals who develop dementia later on [56-58], complicating their use as potential predictors, especially in individuals with cognitive impairment. Indeed, our earlier study indicated that the predictive performance of the CAIDE dementia risk score, developed for long-term prediction based on a midlife risk profile, was limited among memory clinic patients with cognitive complaints [20]. With regard to individual modifiable risk factors, a meta-analysis investigating predictors of progression from MCI to dementia reported a protective effect for higher BMI, which is consistent with our findings [59]. Diabetes and hypertension were also associated with a higher risk of progression to AD dementia, while smoking and hypercholesterolemia were not [59]. In contrast, in a large multicenter memory clinic study of MCI patients [52], hypertension, hypercholesterolemia, diabetes, obesity, and smoking did not influence the rate of cognitive decline and disease progression. This effect did not vary across the International Working Group-2 (IWG-2) and NIA-AA criteria, i.e., it did not seem to depend on the presence or absence of amyloid pathology.

Nevertheless, given the associations we observed between lower BMI, higher SBP, and increased dementia risk in this study, the potential role of modifiable vascular factors in subgroups of memory clinic patients with MCI needs to be further studied. Modifiable vascular/ lifestyle factors could be relevant targets for preventive 
Table 7 Harrell C statistic and model performance in prediction of any dementia/AD dementia (patients with CSF A $42>696$ pg/ $\mathrm{ml})$

\begin{tabular}{|c|c|c|c|c|}
\hline \multirow[t]{2}{*}{ Categories of predictors } & \multirow[t]{2}{*}{ N } & \multirow[t]{2}{*}{ Prediction model } & Dementia & AD dementia \\
\hline & & & \multicolumn{2}{|l|}{ Harrell C } \\
\hline \multirow[t]{7}{*}{ Basic model vs. CSF biomarkers } & \multirow[t]{7}{*}{140} & Age, RAVLT (basic) & 0.74 & 0.74 \\
\hline & & Age, RAVLT, Aß42 & 0.79 & 0.79 \\
\hline & & Age, RAVLT, A 442 , p-tau & 0.80 & 0.82 \\
\hline & & Age, RAVLT, Aß42/p-tau & 0.78 & 0.82 \\
\hline & & Age, RAVLT, Aß42, t-tau & 0.82 & 0.87 \\
\hline & & Age, RAVLT, Aß42/t-tau & 0.81 & 0.86 \\
\hline & & Age, RAVLT, Aß42, p-tau, t-tau & 0.83 & 0.88 \\
\hline \multirow[t]{2}{*}{ Basic model vs. MTA } & \multirow[t]{2}{*}{101} & Age, RAVLT (basic) & 0.74 & 0.75 \\
\hline & & Age, RAVLT, MTA & 0.77 & 0.78 \\
\hline \multirow[t]{2}{*}{ Basic model vs. APOE $\varepsilon 4$ genotype } & \multirow[t]{2}{*}{72} & Age, RAVLT (basic) & 0.73 & 0.71 \\
\hline & & Age, RAVLT, APOE & 0.77 & 0.78 \\
\hline \multirow[t]{2}{*}{ Basic model vs. SBP } & \multirow[t]{2}{*}{109} & Age, RAVLT (basic) & 0.73 & 0.75 \\
\hline & & Age, RAVLT, SBP & 0.77 & 0.78 \\
\hline \multirow[t]{2}{*}{ Basic model vs. BMI } & \multirow[t]{2}{*}{82} & Age, RAVLT (basic) & 0.78 & 0.82 \\
\hline & & Age, RAVLT, BMI & 0.80 & 0.83 \\
\hline \multirow[t]{2}{*}{ Basic model vs. depressive symptoms } & \multirow[t]{2}{*}{105} & Age, RAVLT (basic) & 0.74 & 0.78 \\
\hline & & Age, RAVLT, Cornell score & 0.77 & 0.81 \\
\hline
\end{tabular}

Predictive performance of the prediction models was explored using the Harrell C statistic ( 0.5 indicates no predictive value, whereas 1.0 indicates complete prediction, on a scale from 0 to 1). Comparisons of model performance were conducted between models including the same number of patients: basic model (age, RAVLT delayed recall score) and models expanded with additional predictors. $A D$ Alzheimer's disease, $A P O E$ apolipoprotein $\mathrm{E}, A \beta 42 \beta$-amyloid 1-42, BMI body mass index, CSF cerebrospinal fluid, MTA medial temporal lobe atrophy, visual rating, $p$-tau tau phosphorylated at threonine 181, RAVLT Rey Auditory Verbal Learning Test (delayed recall), SBP systolic blood pressure, $t$-tau total tau

strategies, especially in memory clinic patients with normal A 342 levels who may not be eligible for amyloidfocused pharmacological dementia prevention trials.

\section{Strengths and limitations}

This study included a large sample of well-characterized memory clinic patients with normal CSF A $\beta 42$ levels, which allowed us to investigate a broad range of different biomarkers and clinical characteristics in relation to subsequent dementia development. As data were collected retrospectively from medical records, detailed information on lifestyle factors was not available. Some variables investigated in this study were only available for a subset of patients, potentially limiting the statistical power of the analyses. Due to missing data, some variables were also excluded from the analyses (e.g., cognitive tests for non-memory domains, Fazekas score for white matter lesions).

As in most clinic-based studies, there was considerable variation in follow-up time and number of visits, and due to the fairly short mean follow-up time of approximately 3 years, the possibility that some individuals are slow progressors and develop dementia at a later stage cannot be excluded. Also, potential circularity cannot be fully ruled out, as clinicians were not blinded to CSF biomarkers. However, CSF was routinely collected at the clinic from all referred patients without contraindications for lumbar puncture, not just those with suspected AD pathology. Also, the study population included all patients with a clinical diagnosis of $\mathrm{MCI}$ and normal CSF A 342 as per local cut-offs, regardless of clinicians' notes on MCI subtype or potential underlying causes. Another potential limitation is the upward drift over time in CSF A $\beta 42$ concentrations and cutpoints for normality/abnormality in broadly used assays [33, 34]. To address this issue, we performed sensitivity analyses with a higher cut-off for amyloid positivity and obtained largely comparable results.

Finally, due to differences in patient populations and clinic procedures, our results may not be generalizable to other memory clinics, settings, or populations. As the predictive performance of biomarkers may be influenced by patient age $[12,50,60]$, it is noteworthy that the patient population at the Karolinska University Hospital memory clinic is fairly young (mean age of all examined patients 63 years; in this study 65 years) [61]. The patient population in this study was also highly educated and ethnically relatively homogenous, indicating a need for studies in diverse populations and other geographical settings. 


\section{Conclusions}

Among memory clinic patients with $\mathrm{MCI}$ and normal CSF $A \beta 42$ levels, all three CSF biomarkers $A \beta 42$, $t$-tau, and $p-$ tau were key predictors of progression to dementia/AD dementia. The association between $\mathrm{A} \beta 42$ and clinical progression did not seem to be driven by $A \beta 42$ levels close to the cut-off values. The possibility of underlying AD pathology and risk of progression to dementia should thus not be completely ruled out among MCI patients when A $\beta 42$ levels are within the normal range. While cut-offs may be useful in clinical practice to identify high-risk individuals, establishing an accurate prognosis for individuals with an intermediate risk of progression requires alternative approaches. Simply adjusting the current cut-offs for "normal A $\beta 42$ " may not be the optimal solution, but developing more complex personalized risk prediction tools including continuous CSF biomarkers may be preferable $[50,51]$. In this context, the role of modifiable vascular/lifestyle factors in different subgroups of MCI patients should be further explored.

\section{Supplementary information}

Supplementary information accompanies this paper at https://doi.org/10. 1186/s13195-019-0557-1.

Additional file 1: Table S1. CAIDE risk scores calculated in the present study. Table S2. Baseline characteristics of the study population, by outcome (progression to AD dementia).

\section{Abbreviations}

AD: Alzheimer's disease; APOE: Apolipoprotein E; A $\beta$ : $\beta$-amyloid; $A \beta 42$ : $\beta$ amyloid 1-42; BMl: Body mass index; BP: Blood pressure;

CAIDE: Cardiovascular Risk Factors, Aging and Dementia Study ;

Cl: Confidence interval; CSF: Cerebrospinal fluid; CT: Computed tomography; DSM-IV: Diagnostic and Statistical Manual of Mental Disorders, $4^{\text {th }}$ edition; HR: Hazard ratio; IWG-2: International Working Group-2; MCl: Mild cognitive impairment; MMSE: Mini-Mental State Examination; MRI: Magnetic resonance imaging; MTA: Medial temporal lobe atrophy; NIA-AA: National Institute on Aging-Alzheimer's Association; NINCDS-ADRDA: National Institute of Neurological and Communicative Disorders and Stroke-Alzheimer's Disease and Related Disorders Association; NINDS-AIREN: National Institute of Neurological Disorders and Stroke-Association Internationale pour la Recherche et I'Enseignement en Neurosciences; PET: Positron emission tomography; p-tau: Tau phosphorylated at threonine 181; RAVLT: Rey Auditory Verbal Learning Test; SBP: Systolic blood pressure; t-tau: Total tau

\section{Acknowledgements}

Not applicable.

\section{Authors' contributions}

AR, AS, VJ, NB, and MK conceived and designed the study. AR, AS, VJ, and $\mathrm{GH}$ acquired and analyzed the data. AR, AS, and MK drafted a significant portion of the manuscript and figures. All authors read and approved the final manuscript.

\section{Funding}

The study was supported by Academy of Finland grants 317465, 287490, Finland; Swedish Research Council, Sweden; Alzheimerfonden, Sweden; Region Stockholm (ALF, NSV), Sweden; Knut and Alice Wallenberg Foundation, Sweden; Center for Innovative Medicine (CIMED) at Karolinska Institutet, Sweden; Stiftelsen Stockholms Sjukhem, Sweden; Konung Gustaf V:s och Drottning Victorias Frimurarstiftelse, Sweden; Finnish Cultural Foundation North Savo regional fund, Finland; Finnish Brain Foundation,
Finland; Emil Aaltonen Foundation, Finland; Juho Vainio Foundation, Finland; and European Research Council grant 804371. The funders had no role in the study design; in the collection, analysis, or interpretation of the data; in the preparation of the manuscript; or in the decision to submit the article for publication.

\section{Availability of data and materials}

The datasets generated and/or analyzed during the current study are not publicly available due to ethics legislation in Sweden. For more information, please contact Miia Kivipelto, miia.kivipelto@ki.se

\section{Ethics approval and consent to participate}

The study was approved by the Regional Ethical Review Board in Stockholm, and written informed consent was obtained from all patients.

\section{Consent for publication}

Not applicable.

\section{Competing interests}

The authors declare that they have no competing interests.

\section{Author details}

${ }^{1}$ Department of Neurology, Institute of Clinical Medicine, University of Eastern Finland, Kuopio, Finland. 'Division of Clinical Geriatrics, Centre for Alzheimer Research, Department of Neurobiology, Care Sciences and Society, Karolinska Institutet, Stockholm, Sweden. ${ }^{3}$ Clinic for Cognitive Disorders,

Theme Aging, Karolinska University Hospital-Huddinge, Stockholm, Sweden. ${ }^{4}$ Institute of Public Health and Clinical Nutrition, University of Eastern Finland, Kuopio, Finland. ${ }^{5}$ The Ageing Epidemiology Research Unit, School of Public Health, Imperial College London, London, UK.

Received: 23 August 2019 Accepted: 13 November 2019

Published online: 05 December 2019

\section{References}

1. Petersen RC, Caracciolo B, Brayne C, Gauthier S, Jelic V, Fratiglioni L. Mild cognitive impairment: a concept in evolution. J Intern Med. 2014;275(3): 214-28.

2. Mitchell AJ, Shiri-Feshki M. Rate of progression of mild cognitive impairment to dementia--meta-analysis of 41 robust inception cohort studies. Acta Psychiatr Scand. 2009;119(4):252-65.

3. Dubois B, Feldman HH, Jacova C, Dekosky ST, Barberger-Gateau P, Cummings J, et al. Research criteria for the diagnosis of Alzheimer's disease: revising the NINCDS-ADRDA criteria. Lancet Neurol. 2007;6(8):734-46.

4. Dubois B, Feldman HH, Jacova C, Cummings JL, Dekosky ST, BarbergerGateau P, et al. Revising the definition of Alzheimer's disease: a new lexicon. Lancet Neurol. 2010:9(11):1118-27.

5. Dubois B, Feldman HH, Jacova C, Hampel H, Molinuevo JL, Blennow K, et al. Advancing research diagnostic criteria for Alzheimer's disease: the IWG-2 criteria. Lancet Neurol. 2014;13(6):614-29.

6. Albert MS, DeKosky ST, Dickson D, Dubois B, Feldman HH, Fox NC, et al. The diagnosis of mild cognitive impairment due to Alzheimer's disease: recommendations from the National Institute on Aging-Alzheimer's Association workgroups on diagnostic guidelines for Alzheimer's disease. Alzheimers Dement. 2011;7(3):270-9.

7. Sperling RA, Aisen PS, Beckett LA, Bennett DA, Craft S, Fagan AM, et al. Toward defining the preclinical stages of Alzheimer's disease: recommendations from the National Institute on Aging-Alzheimer's Association workgroups on diagnostic guidelines for Alzheimer's disease. Alzheimers Dement. 2011;7(3):280-92.

8. Jack CR, Albert MS, Knopman DS, McKhann GM, Sperling RA, Carrillo MC, et al. Introduction to the recommendations from the National Institute on Aging-Alzheimer's Association workgroups on diagnostic guidelines for Alzheimer's disease. Alzheimers Dement. 2011;7(3):257-62.

9. Jack CR, Bennett DA, Blennow K, Carrillo MC, Dunn B, Haeberlein SB, et al. NIA-AA research framework: toward a biological definition of Alzheimer's disease. Alzheimers Dement. 2018;14(4):535-62.

10. Jack CR, Knopman DS, Jagust WJ, Shaw LM, Aisen PS, Weiner MW, et al. Hypothetical model of dynamic biomarkers of the Alzheimer's pathological cascade. Lancet Neurol. 2010;9(1):119-28. 
11. Zwan M, van Harten A, Ossenkoppele R, Bouwman F, Teunissen C, Adriaanse $\mathrm{S}$, et al. Concordance between cerebrospinal fluid biomarkers and [11C] PIB PET in a memory clinic cohort. J Alzheimers Dis. 2014; 41(3):801-7.

12. Bertens D, Tijms BM, Scheltens P, Teunissen CE, Visser PJ. Unbiased estimates of cerebrospinal fluid beta-amyloid 1-42 cutoffs in a large memory clinic population. Alzheimers Res Ther. 2017;9(1):8

13. Vos SJ, Verhey F, Frolich L, Kornhuber J, Wiltfang J, Maier W, et al. Prevalence and prognosis of Alzheimer's disease at the mild cognitive impairment stage. Brain. 2015;138(Pt 5):1327-38.

14. Caroli A, Prestia A, Galluzzi S, Ferrari C, van der Flier WM, Ossenkoppele R, et al. Mild cognitive impairment with suspected nonamyloid pathology (SNAP): prediction of progression. Neurology. 2015;84(5):508-15.

15. Alexopoulos P, Werle L, Roesler J, Thierjung N, Gleixner LS, Yakushev I, et al. Conflicting cerebrospinal fluid biomarkers and progression to dementia due to Alzheimer's disease. Alzheimers Res Ther. 2016;8(1):51.

16. Petersen RC, Aisen P, Boeve BF, Geda YE, Ivnik RJ, Knopman DS, et al. Mild cognitive impairment due to Alzheimer disease in the community. Ann Neurol. 2013;74(2):199-208.

17. Prestia A, Caroli A, van der Flier WM, Ossenkoppele R, Van Berckel B, Barkhof $F$, et al. Prediction of dementia in $\mathrm{MCl}$ patients based on core diagnostic markers for Alzheimer disease. Neurology. 2013;80(11):1048-56.

18. Tijms BM, Bertens D, Slot RE, Gouw AA, Teunissen CE, Scheltens P, et al. Low normal cerebrospinal fluid Abeta42 levels predict clinical progression in nondemented subjects. Ann Neurol. 2017;81(5):749-53.

19. Folstein MF, Folstein SE, McHugh PR. "Mini-mental state". A practical method for grading the cognitive state of patients for the clinician. J Psychiatr Res. 1975;12(3):189-98.

20. Enache D, Solomon A, Cavallin L, Kareholt I, Kramberger MG, Aarsland $D$, et al. CAIDE Dementia Risk Score and biomarkers of neurodegeneration in memory clinic patients without dementia. Neurobiol Aging. 2016:42:124-31.

21. Winblad B, Palmer K, Kivipelto M, Jelic V, Fratiglioni L, Wahlund LO, et al. Mild cognitive impairment--beyond controversies, towards a consensus: report of the International Working Group on Mild Cognitive Impairment. J Intern Med. 2004;256(3):240-6.

22. American Psychological Association. Diagnostic and statistical manual of mental disorders. 4th ed. Washington, DC: APA; 1994.

23. McKhann G, Drachman D, Folstein M, Katzman R, Price D, Stadlan EM. Clinical diagnosis of Alzheimer's disease: report of the NINCDS-ADRDA Work Group under the auspices of Department of Health and Human Services Task Force on Alzheimer's Disease. Neurology. 1984;34(7):939-44.

24. Roman GC, Tatemichi TK, Erkinjuntti T, Cummings JL, Masdeu JC, Garcia JH, et al. Vascular dementia: diagnostic criteria for research studies. Report of the NINDS-AIREN International Workshop. Neurology. 1993;43(2):250-60.

25. Neary D, Snowden JS, Gustafson L, Passant U, Stuss D, Black S, et al. Frontotemporal lobar degeneration: a consensus on clinical diagnostic criteria. Neurology. 1998;51(6):1546-54.

26. Emre M, Aarsland D, Brown R, Burn DJ, Duyckaerts C, Mizuno Y, et al. Clinical diagnostic criteria for dementia associated with Parkinson's disease. Mov Disord. 2007;22(12):1689-707.

27. Handels RLH, Vos SJB, Kramberger MG, Jelic V, Blennow K, van Buchem M, et al. Predicting progression to dementia in persons with mild cognitive impairment using cerebrospinal fluid markers. Alzheimers Dement. 2017; 13(8):903-12.

28. Religa D, Fereshtehnejad SM, Cermakova P, Edlund AK, Garcia-Ptacek S, Granqvist N, et al. SveDem, the Swedish Dementia Registry - a tool for improving the quality of diagnostics, treatment and care of dementia patients in clinical practice. PLoS One. 2015;10(2):e0116538.

29. Kramberger $M G$, Jelic $V$, Kareholt I, Enache $D$, Eriksdotter Jonhagen $M$, Winblad B, et al. Cerebrospinal fluid Alzheimer markers in depressed elderly subjects with and without Alzheimer's disease. Dement Geriatr Cogn Dis Extra. 2012;2(1):48-56.

30. Garcia-Ptacek S, Cavallin L, Kareholt I, Kramberger MG, Winblad B, Jelic V, et al. Subjective cognitive impairment subjects in our clinical practice. Dement Geriatr Cogn Dis Extra. 2014:4(3):419-30.

31. Miley-Akerstedt A, Jelic V, Marklund K, Walles H, Akerstedt T, Hagman G, et al. Lifestyle factors are important contributors to subjective memory complaints among patients without objective memory impairment or positive neurochemical biomarkers for Alzheimer's disease. Dement Geriatr Cogn Dis Extra. 2018;8(3):439-52.
32. Toschi N, Lista S, Baldacci F, Cavedo E, Zetterberg H, Blennow K, et al. Biomarker-guided clustering of Alzheimer's disease clinical syndromes. Neurobiol Aging. 2019;83:42-53.

33. Tijms BM, Willemse EAJ, Zwan MD, Mulder SD, Visser PJ, van Berckel BNM, et al. Unbiased approach to counteract upward drift in cerebrospinal fluid amyloid-beta 1-42 analysis results. Clin Chem. 2018; 64(3):576-85

34. Schindler SE, Sutphen CL, Teunissen C, McCue LM, Morris JC, Holtzman DM, et al. Upward drift in cerebrospinal fluid amyloid beta 42 assay values for more than 10 years. Alzheimers Dement. 2018;14(1):62-70.

35. Scheltens $P$, Leys $D$, Barkhof F, Huglo D, Weinstein HC, Vermersch $P$, et al. Atrophy of medial temporal lobes on MRI in "probable" Alzheimer's disease and normal ageing: diagnostic value and neuropsychological correlates. J Neurol Neurosurg Psychiatry. 1992;55(10):967-72.

36. Rosenberg SJ, Ryan JJ, Prifitera A. Rey Auditory-Verbal Learning Test performance of patients with and without memory impairment. J Clin Psychol. 1984;40(3):785-7.

37. Alexopoulos GS, Abrams RC, Young RC, Shamoian CA. Cornell scale for depression in dementia. Biol Psychiatry. 1988;23(3):271-84.

38. Kivipelto M, Ngandu T, Laatikainen T, Winblad B, Soininen H, Tuomilehto J. Risk score for the prediction of dementia risk in 20 years among middle aged people: a longitudinal, population-based study. Lancet Neurol. 2006; 5(9):735-41

39. Exalto LG, Quesenberry CP, Barnes D, Kivipelto M, Biessels GJ, Whitmer RA. Midlife risk score for the prediction of dementia four decades later. Alzheimers Dement. 2014:10(5):562-70.

40. Jack CR, Knopman DS, Chetelat G, Dickson D, Fagan AM, Frisoni GB, et al. Suspected non-Alzheimer disease pathophysiology--concept and controversy. Nat Rev Neurol. 2016;12(2):117-24.

41. Abner EL, Kryscio RJ, Schmitt FA, Fardo DW, Moga DC, Ighodaro ET, et al. Outcomes after diagnosis of mild cognitive impairment in a large autopsy series. Ann Neurol. 2017:81(4):549-59.

42. Nelson PT, Dickson DW, Trojanowski JQ, Jack CR, Boyle PA, Arfanakis K, et al. Limbic-predominant age-related TDP-43 encephalopathy (LATE): consensus working group report. Brain. 2019;142(6):1503-27.

43. Stern Y. Cognitive reserve in ageing and Alzheimer's disease. Lancet Neurol. 2012;11(11):1006-12.

44. Jack CR, Wiste HJ, Vemuri P, Weigand SD, Senjem ML, Zeng G, et al. Brain beta-amyloid measures and magnetic resonance imaging atrophy both predict time-to-progression from mild cognitive impairment to Alzheimer's disease. Brain. 2010;133(11):3336-48.

45. Farrell ME, Kennedy KM, Rodrigue KM, Wig G, Bischof GN, Rieck JR, et al. Association of longitudinal cognitive decline with amyloid burden in middle-aged and older adults: evidence for a dose-response relationship. JAMA Neurol. 2017;74(7):830-8.

46. Farrell ME, Chen X, Rundle MM, Chan MY, Wig GS, Park DC. Regional amyloid accumulation and cognitive decline in initially amyloid-negative adults. Neurology. 2018;91(19):e1809-21.

47. Landau SM, Horng A, Jagust WJ, Alzheimer's Disease Neuroimaging Initiative. Memory decline accompanies subthreshold amyloid accumulation. Neurology. 2018;90(17):e1452-60.

48. Vos SJ, van Rossum IA, Verhey F, Knol DL, Soininen H, Wahlund LO, et al. Prediction of Alzheimer disease in subjects with amnestic and nonamnestic MCl. Neurology. 2013;80(12):1124-32.

49. Elias-Sonnenschein LS, Viechtbauer W, Ramakers IH, Verhey FR, Visser PJ. Predictive value of APOE-epsilon4 allele for progression from $\mathrm{MCI}$ to $\mathrm{AD}$ type dementia: a meta-analysis. J Neurol Neurosurg Psychiatry. 2011;82(10): 1149-56.

50. van Maurik IS, Zwan MD, Tijms BM, Bouwman FH, Teunissen CE, Scheltens $P$, et al. Interpreting biomarker results in individual patients with mild cognitive impairment in the Alzheimer's biomarkers in daily practice (ABIDE) project. JAMA Neurol. 2017;74(12):1481-91.

51. van Maurik IS, Vos SJ, Bos I, Bouwman FH, Teunissen CE, Scheltens P, et al. Biomarker-based prognosis for people with mild cognitive impairment (ABIDE): a modelling study. Lancet Neurol. 2019;18(11):1034-44.

52. Bos I, Vos SJ, Frolich L, Kornhuber J, Wiltfang J, Maier W, et al. The frequency and influence of dementia risk factors in prodromal Alzheimer's disease. Neurobiol Aging. 2017;56:33-40.

53. Kivipelto M, Ngandu T, Fratiglioni L, Viitanen M, Kareholt I, Winblad B, et al. Obesity and vascular risk factors at midlife and the risk of dementia and Alzheimer disease. Arch Neurol. 2005;62(10):1556-60. 
54. Legdeur N, Heymans MW, Comijs HC, Huisman M, Maier AB, Visser PJ. Age dependency of risk factors for cognitive decline. BMC Geriatr. 2018;18(1):187.

55. Solomon A, Mangialasche F, Richard E, Andrieu S, Bennett DA, Breteler M, et al. Advances in the prevention of Alzheimer's disease and dementia. Intern Med. 2014;275(3):229-50.

56. Solomon A, Kareholt I, Ngandu T, Winblad B, Nissinen A, Tuomilehto J, et al. Serum cholesterol changes after midlife and late-life cognition: twenty-oneyear follow-up study. Neurology. 2007;68(10):751-6.

57. McGrath ER, Beiser AS, DeCarli C, Plourde KL, Vasan RS, Greenberg SM, et al. Blood pressure from mid- to late life and risk of incident dementia. Neurology. 2017;89(24):2447-54.

58. Buchman AS, Wilson RS, Bienias JL, Shah RC, Evans DA, Bennett DA. Change in body mass index and risk of incident Alzheimer disease. Neurology. 2005: 65(6):892-7.

59. Li JQ, Tan L, Wang HF, Tan MS, Tan L, Xu W, et al. Risk factors for predicting progression from mild cognitive impairment to Alzheimer's disease: a systematic review and meta-analysis of cohort studies. J Neurol Neurosurg Psychiatry. 2016;87(5):476-84

60. Mattsson N, Rosen E, Hansson O, Andreasen N, Parnetti L, Jonsson M, et al. Age and diagnostic performance of Alzheimer disease CSF biomarkers. Neurology. 2012;78(7):468-76.

61. Andersson C. Predictors of cognitive decline in memory clinic patients. Karolinska Institutet; 2007.

\section{Publisher's Note}

Springer Nature remains neutral with regard to jurisdictional claims in published maps and institutional affiliations.

Ready to submit your research? Choose BMC and benefit from:

- fast, convenient online submission

- thorough peer review by experienced researchers in your field

- rapid publication on acceptance

- support for research data, including large and complex data types

- gold Open Access which fosters wider collaboration and increased citations

- maximum visibility for your research: over $100 \mathrm{M}$ website views per year

At $\mathrm{BMC}$, research is always in progress.

Learn more biomedcentral.com/submissions 